\title{
Is cone-beam computed tomography an essential diagnostic tool for endodontic practice?
}

\begin{abstract}
One of the major limitations of traditional periapical radiography is its two-dimensional representation of three-dimensional structures. It is proposed, quite rationally, that the ability to evaluate an area of interest in three dimensions would be of great diagnostic value in endodontics. The advent of cone beam computed tomography (CBCT) appears to offer this as a realistic possibility for practitioners. However, all diagnostic and therapeutic interventions must be evidence-based, so we must consider whether this new imaging modality is appropriate for the purpose of endodontic diagnosis. As CBCT has been in use in dentistry since 1998, a considerable body of literature regarding its use is now available. CBCT shows promise in improving diagnosis of apical periodontitis, resorbtive lesions, and detection of root canals and in pre-surgical planning. Several shortcomings have also been highlighted however, such as poor image quality if there is patient movement, artefact production, poor resolution, cost and training requirements. As of now, its use can be recommended as good practice in very select cases, such as pre-surgical assessment where proximity to important structures is a concern. As of yet however, there remains a paucity of evidence for its routine use in clinical endodontic situations due to insufficient quality research. It is commonly unjustifiable due to the increase in radiation exposure over traditional radiography and there is a significant capital investment required to purchase one of these machines in return for limited evidence-based use. For now, cone beam computed tomography cannot be deemed an essential diagnostic tool for endodontic practice.
\end{abstract}

Keywords: cone beam computed tomography, CBCT, endodontic, 3d, dental imaging; radiography
Volume 9 Issue I - 2018

\author{
Joey Donovan ${ }^{1,2}$ \\ 'Orange Orthodontics \& Dentofacial Orthopaedics Pte Ltd, \\ Singapore \\ ${ }^{2}$ School of Health Sciences, Nanyang Polytechnic, Singapore
}

Correspondence: Joey Donovan, Orange Orthodontics \& Dentofacial Orthopaedics Pte Ltd, Lucky Plaza Suite \#05-44, 304 Orchard Road, 238863, Singapore, Tel +65-67370544, Fax+65-67360543, Email drjod@gmail.com

Received: December II, 2017 | Published: January II, 2018

\section{Introduction}

It is with a certain degree of trepidation that this essayist begins, knowing the weight of evidence that is required to declare that something is essential. Can Cone Beam Computed Tomography (CBCT) really break free from the lowly ranks of the useful, and join the upper echelons of the absolutely necessary? If so, it seems as though endodontic treatment to this point, which has been carried out without this technology since the early $20^{\text {th }}$ century, would be dishonourably discharged to the title of little more than pre-extraction treatment. From the outset, this would seem like an unfair dismissal indeed, but nevertheless, the evidence needs to be examined.

\section{Background}

With the exception of digitalisation, dental radiography has remained largely unaltered, using essentially the same $2 \mathrm{D}$ intraoral imaging technique that Otto Walkhoff pioneered in $1896 .{ }^{1}$ Typically, endodontic treatment involves a minimum of five radiographs. The first is diagnostic, in conjunction with history, examination and other special tests to evaluate if a tooth requires, and is suitable for, endodontic treatment. Subsequently, intraoperative radiographs establish working length of the root canal, correct placement of the master gutta percha point, and assess the final obturation of the canal. ${ }^{2}$ Afterwards, radiographs are used months following treatment to assess bone healing and thereby evaluate the success of the endodontic treatment carried out. ${ }^{3}$ A traditional periapical radiograph however, is a two dimensional representation of a three dimensional structure, which has the obvious limitation of being unable to accurately portray certain morphological and biological features due to superimposition of structures. ${ }^{4}$ Perhaps the greatest limitation of traditional dental imaging is the 'flattening' of the image, which precludes the viewer from visualising structures in the third (buccolingual) dimension. ${ }^{5}$ Overlying structures such as cortical bone, sinuses and trabeculae can become superimposed on the area of interest, complicating the interpretation of the radiograph. ${ }^{5}$ The principal of parallax is employed frequently in 2D dental imaging to try and overcome this inherent flaw, where a second radiograph is taken at an angle to the first, giving a perception of depth when structures move relative to one another. However, several studies have concluded that multiple radiographs using this principal often do not reveal more than a single exposure, nor do they guarantee correct identification of the relevant anatomy. ${ }^{6,7}$ It is proposed, quite rationally, that the ability to evaluate an area of interest in three dimensions would be of great diagnostic value in endodontics. ${ }^{8}$ CBCT, first used in dentistry in 1998, has emerged as one of the best methods to obtain a 3D image for dental purposes. The European commission financed the SEDENTEXCT project to develop an evidenced-based framework for CBCT use. In this image modality, the x-ray beam is in the shape of a cone, which rotates around the patients head, capturing a spherical or cylindrical volume of data, called a field of view. ${ }^{7}$ A composite image is composed of $3 \mathrm{~d}$ pixels, called voxels. Voxels rendered from CBCT are isotropic, which means they are equal in length, height and depth and can therefore be viewed from every possible angle with precise geometrical accuracy that has been confirmed in several studies..$^{9-11}$ 


\section{Aims and objectives}

1. Does CBCT offer any additional benefit to patients in comparison to traditional radiography for endodontic purposes?

2. If so, are these advantages so great as to justify pronouncing the technology as essential to modern endodontic practice?

3. To discuss the relevant information about the radiation exposure and associated risks of $\mathrm{CBCT}$ versus traditional endodontic radiology.

4. To discuss the pragmatism of introducing this technology into endodontic practices under the heading of size, cost and ease of use of the hardware and software.

\section{Methods}

Searches were performed in The Cochrane Library, Science Direct, Google Scholar, PubMed and the UCC online journal database (available at booleweb.ucc.ie). Internet searches were also performed.

\section{Review of the literature}

\section{Suggested advantages of CBCT}

Detection of apical periodontitis: The early detection of periapical lesions is vital to successful endodontic practice, with success of endodontic treatment increased if root treatment is carried out before radiographic changes have taken place. ${ }^{12} \mathrm{CBCT}$ can detect radiolucent periapical lesions before they appear on traditional radiographs. ${ }^{11}$ Some studies have suggested that CBCT represents the "Gold Standard" in diagnosis of periapical periodontitis, as it has shown $100 \%$ sensitivity and specificity in detecting these lesions in in vitro trials. ${ }^{11}$ In a study by Durack \& Patel $^{5}$ it was suggested that if clinical symptoms and signs of a periapical infection were present, but inconclusive on conventional radiographs, then CBCT "may prove a useful adjunct" to the diagnosis of endodontic disease. ${ }^{5}$

Detection of resorptive lesions: Several studies have been conducted to compare the ability of $\mathrm{CBCT}$ to detect internal and external inflammatory resorption. Root resorption is the loss of dental hard tissue due to the activity of odontoclastic cells, which can be exacerbated by endodontic infection. ${ }^{13}$ Thus, it is vital to diagnose it correctly in order to carry out appropriate treatment. Patel et al. ${ }^{14}$ conducted a study in which they compared the diagnoses and treatment plans of six examiners, who were presented with both $\mathrm{CBCT}$ radiographs and traditional periapical radiographs using a paralleling technique. Intraoral radiography was deemed "reasonably accurate" in correctly diagnosing internal and external root resorption, correctly identifying lesions in $78 \%$ and $83 \%$ of cases respectively. When CBCT scans were used, however, the resorptive defects were diagnosed with $100 \%$ accuracy, which was concluded to be as a result of the ability to assess the lesions in three-dimensions. Interestingly, while the presence and type of root resorption was elucidated with $100 \%$ accuracy with $\mathrm{CBCT}$, the correct course of treatment was chosen in only $80 \%$ of cases, highlighting the fact that no diagnostic tool by itself is a silver bullet. ${ }^{15}$

Detection of root canals/root morphology: Successful root treatment depends largely on pre-operative planning and knowledge of the number, location, and shape of a root canal system. There are a large numbers of case reports (eg [8] Case \#1) where the reason for failure of previous root canal treatment has been identified as a failure of conventional radiography to disclose the number of canals in a tooth. Several studies have been conducted which were in agreement that extra canals were more frequently identified, along with their exact locations, when CBCT was used over conventional radiography. ${ }^{5}$ This would allow the endodontist to design the most appropriate access cavity on a case-by-case basis. ${ }^{5}$ Estrela et al describe a method of determining the root canal radius using software to manipulate CBCT images, enabling the dental practitioner to choose the most appropriate endodontic instruments and instrumentation technique to debride and shape the canal. ${ }^{4}$ Such information is not available from two-dimensional periapical or panoramic radiographs.

Pre-surgical planning: Several authors have recognised the potential value of $\mathrm{CBCT}$ in planning endodontic surgery. ${ }^{11-16}$ It is suggested that important pre-operative information such as the thickness of the cortical plate, location of the sinus and its relationship to the teeth, inclination of roots of teeth as well as the exact location of the lesion can be gleaned from CBCT images. ${ }^{11}$ Velvart et al. (2001) extrapolated data from a Medical Computed Tomography study which found that medical CT identified the spatial relationship between root apices and the Inferior Dental Canal in all cases, compared to $40 \%$ of cases measured correctly using traditional radiography. Another study found traditional intraoral radiographs to be less sensitive than CBCT for localising lesions, especially in instances of maxillary molars where the maxillary sinus was in close proximity to the root in question. ${ }^{17}$ Scarfe et al.$^{18}$ reported an exciting application of 3-dimensional CBCT images, that of $3 \mathrm{D}$ printing or stereolithography which would enable dental practitioners to create a tangible, true-scale model of the case. ${ }^{18}$

Post-operatively: Several studies have highlighted the shortcomings of using traditional radiography to evaluate the success of endodontic treatment, and propose CBCT in its place. It is claimed that success rates quoted for endodontic treatment since 1922 are likely to have been an overestimation as periapical radiographs were used as the diagnostic tool. ${ }^{3}$ That particular study concluded that a large number of cases which were confirmed healthy by periapical radiograph displayed signs of apical periodontitis with CBCT or histology. This corroborated the clinical work of Liang et al., ${ }^{19}$ who found success rates to be lower when measured using $\mathrm{CBCT}$ versus conventional radiograph after 2 years. ${ }^{19}$ On the basis of these and other studies, it has been questioned whether the Quality Guidelines for Endodontic treatment may have to be revised to include stricter evaluation of healing using $\mathrm{CBCT}^{5-20}$ Other longitudinal studies, however, have shown that healing which is evident on a periapical radiograph is valid with a "high degree of confidence". ${ }^{21,22}$

Accurate temporal perspective: Endodontic lesions require time to heal post-operatively before they are assessed for long term success or failure. In a study by Rudolph \& White, ${ }^{23}$ it was found that serial radiographs taken using traditional radiography will show inconsistencies due to changes in x-ray beam or film orientation, even with the use of alignment techniques, such as preparing bite blocks to minimise these discrepancies. ${ }^{23}$ It has been shown that these slight variations in angulation can result in the lesion showing definite signs of healing, when in fact the lesion has remained the same size. ${ }^{7} \mathrm{CBCT}$, due to its isotropic voxels and confirmed geometric accuracy in all dimensions, does not rely on replication of patient or beam angulation, as images can be aligned and compared in all planes using software. ${ }^{10}$

Patient comfort when planning Endodontic Treatment after trauma: Trauma is one reason why a tooth may need to be endodontically treated. In these cases, the patient may be in severe 
discomfort as a result of the trauma, and may have difficulty accommodating the bulky intraoral film and film-holder used in traditional radiography. As CBCT is an extra-oral imaging technique, it does not require an intraoral film, and so is a more comfortable method of planning endodontic treatment for these patients. ${ }^{5}$

\section{Disadvantages of CBCT}

Requires additional training: The literature is at odds as to the level of additional training required to operate and analyse the data from CBCT machines effectively. Patel et al (2009) stated that "users of CBCT must be adequately trained in CBCT radiology as well as interpretation of these images as they are completely different from conventional radiography systems". ${ }^{11}$ By contrast, another study found no difference in the results obtained from reading CBCT images between examiners with varying levels of experience from post-graduate to specialist level. ${ }^{15}$

\section{Image quality}

Resolution: Currently, CBCT has inferior image resolution to conventional radiographs, with the latter having an order of magnitude more line pairs per millimetre in comparison to CBCT (Patel, 2009).

Movement: In vitro studies do not involve any movement of the object being imaged. This is imperative for geometrical accuracy of CBCT images, which require the patient to remain absolutely still for the duration of the scan, as motion leads to image degradation. ${ }^{1}$ This has been found to be problematic in vivo, due to the lengthy scan times of 15-40 seconds for this image modality. ${ }^{11}$

Beam hardening and scatter: This is a phenomenon inherent in CBCT imaging, where preferential absorption of low energy photons by dense materials leads to an increase in mean photon energy of the $\mathrm{x}$-ray beam, resulting in 'hardening' and artefact production in the image. ${ }^{2}$ This is one of the most significant drawbacks of CBCT which can affect the "quality and diagnostic accuracy" of the images obtained. If the area of interest is near a dense structure such as enamel or a neighbouring restoration or metal, the images diagnostic value may be negligible. ${ }^{4-25}$ In addition, CBCT has been found to be inferior to conventional radiography when it comes to judging the quality of root filling due to the streaking artefacts produced. ${ }^{24}$

\section{Radiation Considerations}

Radiation detriment can be considered as the total harm experienced by an irradiated individual including the nominal increase in risk of cancer and hereditable effects. The Irish Dental Council states that "The dental exposure shall show a sufficient net benefit compared with any detriment that exposure might cause. The radiation dose shall be 'as low as is reasonably achievable' for the aims of the radiographic examination being undertaken". ${ }^{26}$ Applying this ALARA principal, the fact that $\mathrm{CBCT}$ exposes the patient to higher levels of ionising radiation than conventional radiography should restrict its use to those cases where conventional radiographs do not provide sufficient information to make an informed clinical decision. ${ }^{27} \mathrm{CBCT}$ units have a huge array of variables, for example the size of the field of view can be reduced to decrease exposure, or resolution can be increased to increase detail in images, but at the expense of increased radiation dose to name but a few. ${ }^{11}$ For this reason, the average effective radiation dose is difficult to determine, but any CBCT scan is at very minimum the equivalent of three conventional periapical exposures..$^{28}$ Despite the fact that the field of view in the majority of $\mathrm{CBCT}$ machines can be adjusted, the minimum size is $4 \mathrm{~cm} \times 4 \mathrm{~cm} .{ }^{5}$ As many endodontic procedures require the treatment of only one tooth, even the minimum FOV exposes the patient to unnecessary radiation. ${ }^{29}$

\section{Practical considerations}

Cost: CBCT scanners are composed of relatively simple hardware, consisting of an X-ray source and a detector, and low-cost computers are available which can render the 3D images. This, coupled with an increasingly competitive market for dental hardware has made these machines more affordable for modern endodontic practices, resulting in an increase in their uptake. ${ }^{30}$

Size: Unlike medical CT scanners, CBCT captures images with the patient in an upright position. This allows CBCT machines to occupy a similar footprint to panoramic imaging machines, which makes them suitable for dental practice. ${ }^{11}$

\section{Discussion}

Any decision on policy in dentistry should always be evidence based. The evidence base in support of a CBCT as an essential diagnostic tool is underwhelming. Theoretically at least, CBCT has many advantages over traditional radiography. A large number of studies have shown its superiority in diagnosis of apical periodontitis, resorbtive lesions, and detection of root canals and in pre-surgical planning. ${ }^{11-15}$ Many of these studies, however, suffer from small sample sizes, reference standards based on consensus committees rather than objective measurements, or lack of compatibility with genuine clinical scenarios. There is a distinct imbalance in the literature between numbers of in vitro and clinical trials, which has led this essayist to query whether many of the results obtained can truly be extrapolated to clinical settings. The literature reveals that in vitro trials eliminate many of CBCT's clinical shortcomings, that of patient movement degrading the image quality, and individual patient's restorations creating image artefacts. ${ }^{1-28}$ The three dimensional nature of the image is no doubt favourable, but the low resolution of CBCT images is a matter of concern, especially in an area of dental practice like endodontics where fine detail is a requisite. ${ }^{11}$ The case for CBCT is strongest in the planning phase of endodontic treatment, with little recommendation for intra-operative use, and some evidence to suggest that $\mathrm{CBCT}$ may in fact be inferior post operatively due to streaking artefacts from root filling materials. ${ }^{27} \mathrm{CBCT}$ may also be tentatively endorsed for planning of surgical endodontic procedures where there is a risk due to proximity of important structures. The advent of stereolithography may mean $\mathrm{CBCT}$ might have a major application in the future for training purposes or for surgeons to familiarise themselves with a particular case pre-operatively. ${ }^{17}$ One promising aspect of this technology, that of long-term assessment of treatment outcome, should be further explored. Its use, however, should be primarily in research studies at this point, to evaluate the accuracy of current methods of assessment of treatment outcome with a view to updating outcome assessment guidelines if deemed necessary. ${ }^{3}$ Most papers reviewed, while advocating CBCT's advantages, have stressed that its use should be limited to an adjunctive diagnostic tool in those cases for which conventional radiography gives inadequate information. ${ }^{29}$ In the vast majority of cases, the benefit to patients is minimal and does not justify the increased ionising radiation exposure of a CBCT scan. Ionising radiation exposure is highlighted as a central issue to this decision. For routine diagnostic use, CBCT's advantages fall short of the Irish Dental Councils ionising radiation guidelines, which demand that any increase in radiation be exceeded by an 
increase in patient benefit. ${ }^{26}$ Despite adjustable settings on most CBCT machines, to achieve acceptable resolutions the radiation exposure must be increased. ${ }^{11}$ In addition, the field of view cannot be reduced to a single tooth area, which would be acceptable for most endodontic procedures. For this reason, radiation exposure from CBCT is a multiple of that from conventional radiography. ${ }^{28}$ Without achieving significantly greater patient benefit or significantly reducing radiation levels through improved technology, it is unlikely that CBCT will be deemed acceptable, let alone essential to endodontic practice. As with all dental procedures, the patient is the foremost concern. However, dental practices also have to be economically viable. From a practical point of view, while CBCT machines are not disproportionately expensive, they do require a significant capital investment. In addition to this, because of the relative youth of the technology, depreciation and upgrade requirements as the technology improves will be greater for this type of machine than traditional radiography equipment. ${ }^{30}$

\section{Conclusion}

The battle is lost, but the war is by no means over. CBCT is a highly promising technology that will in all likelihood be used increasingly in dentistry and in the field of endodontics in particular as the technology and quality of research in the area improves.

1. In very select cases, its use can be recommended as good practice, such as pre-surgical assessment where proximity to important structures is a concern. As of yet however, there remains a paucity of evidence for its routine use in clinical endodontic situations due to insufficient quality research.

2. $\mathrm{CBCT}$ is commonly unjustifiable due to the increase in radiation exposure over traditional radiography.

3. There is a significant capital investment required to purchase one of these machines in return for limited evidence-based use.

4. For the reasons outlined above, cone beam computed tomography cannot be deemed an essential diagnostic tool for endodontic practice.

\section{Funding details}

None.

\section{Acknowledgments}

None.

\section{Conflicts of interest}

The author declares that there is no conflict of interest.

\section{References}

1. Tyndall DA, Rathore S. Cone-beam CT diagnostic applications: caries, periodontal bone assessment, and endodontic applications. Dent Clin North Am. 2008;52(4):825-841.

2. Scarfe WC, Levin MD, Gane D, et al. Use of cone beam computed tomography in endodontics. Int J Dent. 2009;634567.

3. Wu MK, Shemesh H, Wesselink PR. Limitations of previously published systematic reviews evaluating the outcome of endodontic treatment. Int Endod J. 2009;42(8):656-666.

4. Estrela C, Bueno MR, Sousa-Neto MD, et al. Method for determination of root curvature radius using cone-beam computed tomography images. Braz Dent J. 2008;19(2):114-118.
5. Durack C, Patel S. Cone beam computed tomography in endodontics. Brazilian Dental Journal. 2012;23(3):179-191.

6. Barton DJ, Clark SJ, Eleazer PD, et al. Tuned-aperture computed tomography versus parallax analog and digital radiographic images in detecting second mesiobuccal canals in maxillary first molars. Oral Surg Oral Med Oral Pathol Oral Radiol Endod. 2003;96(2):223-228.

7. Patel S, Dawood A, Whaites E, et al. New dimensions in endodontic imaging: part 1. Conventional and alternative radiographic systems. Int Endod J. 2009;42(6):447-462.

8. Cotton TP, Geisler TM, Holden DT, et al. Endodontic applications of cone-beam volumetric tomography. J Endod. 2007;33(9):1121-1132.

9. Patel S, Dawood A, Ford TP, et al. The potential applications of cone beam computed tomography in the management of endodontic problems. Int Endod J. 2007;40(10):818-830.

10. Kobayashi K, Shimoda S, Nakagawa Y, et al. Accuracy in measurement of distance using limited cone-beam computerized tomography. Int J Oral Maxillofac Implants. 2004;19(2):228-331.

11. Patel S. New dimensions in endodontic imaging: Part 2. Cone beam computed tomography. Int Endod J. 2009;42(6):463-475.

12. Friedman S. Prognosis of initial endodontic therapy. Endodontic Topics. 2002;2:59-88.

13. Gunraj MN. Dental root resorption. Oral Surgery, Oral Medicine, Oral Pathology, Oral Radiology, and Endodontology. 1999;88(6):647-653.

14. Patel S, Dawood A, Mannocci F, et al. Detection of periapical bone defects in human jaws using cone beam computed tomography and intraoral radiography. Int Endod J. 2009a;42(6):507-515.

15. Patel S, Dawood A, Wilson R, et al. The detection and management of root resorption lesions using intraoral radiography and cone beam computed tomography - an in vivo investigation. Int Endod J. 2009c;42(9):831-838.

16. Rigolone M, Pasqualini D, Bianchi L, et al. Vestibular surgical access to the palatine root of the superior first molar: "low-dose cone-beam" CT analysis of the pathway and its anatomic variations. $J$ Endod. 2003;29(11):773-775.

17. Low KM, Dula K, Burgin W, et al. Comparison of periapical radiography and limited cone-beam tomography in posterior maxillary teeth referred for apical surgery. J Endod. 2008;34(5):557-562.

18. Scarfe WC, Farman AG, Sukovic P. Clinical applications of conebeam computed tomography in dental practice. J Can Dent Assoc. 2006;72(1):75-80.

19. Liang YH, Li G, Wesselink PR, et al. Endodontic outcome predictors identified with periapical radiographs and cone-beam computed tomography scans. $J$ Endod. 2010;37(3):326-331.

20. European Society of Endodontology. Quality guidelines for endodontic treatment: consensus report of the European Society of Endodontology. Int Endod J. 2006;39(12):921-930.

21. Fristad I, Molven O, Halse A. Nonsurgically retreated root filled teethradiographic findings after 20-27 years. Int Endod J. 2004;37(1):12-18.

22. Mead C, Javidan-Nejad S, Mego ME, et al. Levels of Evidence for the Outcome of Endodontic Surgery. Journal of endodontics. 2005;31(1):19-24.

23. Rudolph DJ, White SC. Film-holding instruments for intraoral subtraction radiography. Oral Surg Oral Med Oral Pathol. 1998;65(6):767-772.

24. Sogur E, Baksi BG, Grondahl HG. Imaging of root canal fillings: a comparison of subjective image quality between limited cone-beam CT, storage phosphor and film radiography. Int Endod J. 2007;40(3):179-185. 
25. Lofthag-Hansen S, Huumonen S, Grondahl K, et al. Limited cone-beam CT and intraoral radiography for the diagnosis of periapical pathology. Oral Surg Oral Med Oral Pathol Oral Radiol Endod. 2007;103(1):114-119.

26. Irish Dental Council. Protocols for Standard Radiological Practice, Ireland. 2010.

27. Fanning B. CBCT--the justification process, audit and review of the recent literature. J Ir Dent Assoc. 2012;57(5):256-261.
28. Arai Y, Honda K, Iwai K, et al. Practical model "3DX" of limited cone-beam X-ray CT for dental use. International Congress Series. 2001;1230:713-718.

29. European Commission. Cone Beam CT for Dental and Maxillofacial Radiology. Evidence Based Guidelines, USA; 2012.

30. Arnheiter C, Scarfe WC, Farman AG. Trends in maxillofacial cone-beam computed tomography usage. Oral Radiology. 2006;22(2):80-85. 